\title{
La France et l'Autriche-Hongrie : de l'Europe des dynasties à celle des États-nations
}

Frankreich und Österreich-Ungarn, vom dynastischen Europa zu den Nationalstaaten

France and Austria-Hungary, from Dynastic Europe to National States

\section{François Genton}

\section{CpenEdition}

\section{Journals}

Édition électronique

URL : http://journals.openedition.org/austriaca/290

DOI : 10.4000/austriaca. 290

ISSN : 2729-0603

\section{Éditeur}

Presses universitaires de Rouen et du Havre

\section{Édition imprimée}

Date de publication : 1 décembre 2018

Pagination : 17-38

ISBN : 979-10-240-1354-1

ISSN : 0396-4590

\section{Référence électronique}

François Genton, « La France et l'Autriche-Hongrie : de l'Europe des dynasties à celle des Étatsnations », Austriaca [En ligne], 87 | 2018, mis en ligne le 01 mars 2020, consulté le 28 janvier 2021. URL : http://journals.openedition.org/austriaca/290; DOI : https://doi.org/10.4000/austriaca.290 


\section{La France et l'Autriche-Hongrie : de l'Europe des dynasties à celle des États-nations}

Cette étude tente d'abord d'esquisser un rapide tableau des idées toutes faites qui concernent la première guerre mondiale et ses résultats aujourd'hui dans différents pays et les confronte aux discussions, mais aussi à un certain consensus dominant dans la recherche historiographique actuelle. Elle s'intéresse ensuite à la spécificité de la situation mémorielle française, dans laquelle le souvenir de la Grande Guerre reste très vivant, et tente d'identifier les différents facteurs qui peuvent expliquer ce fait. L'un de ces facteurs est au cœur de l'étude, à savoir la pérennité d'un souvenir, vague, mais toujours présent, et capable de résister à l'accumulation de ressentis négatifs relatifs à la guerre et aux périodes qui suivirent. Ce souvenir lié à la représentation d'une guerre qui a durablement changé le monde et notamment l'Europe pourrait bien mettre en cause une chronologie qui s'est imposée depuis plusieurs décennies et qui fait commencer le " court $\mathrm{xx}^{\mathrm{e}}$ siècle " en 1914, ce qui condamne à faire de l'année 1918 et des traités qui suivirent un épisode parmi d'autres dans une histoire qui ne se termine vraiment qu'en 1989-1991 avec la fin de la guerre froide. L'ambition de cette étude est de s'opposer à cette vision et de rendre à l'ordre européen, politique et territorial, instauré à la fin de la Grande Guerre, la signification historique positive et constructive qui lui revient. Dans cette perspective, le problème de l'Autriche-Hongrie occupe le premier rang. Cet État n'avait certainement pas d'un point de vue politique tous les défauts que lui attribua la propagande de l'Entente victorieuse et des élites politiques des États nés du démantèlement, mais à la fin d'un conflit particulièrement meurtrier, sa survie, sous quelque forme que ce soit, était exclue de l'espace des possibles. Elle était simplement inenvisageable parce que l'Europe tournait la page multiséculaire de l' « ordre » dynastique pour ouvrir celle de l'organisation du continent en États-nations dont les régimes devaient être fondés sur la souveraineté du peuple et la démocratie. Ce projet a certes commencé par échouer en 
grande partie, un échec qui marque l'histoire du court $\mathrm{xx}^{\mathrm{e}}$ siècle, mais il s'est montré capable d'une résilience que les nostalgiques des empires disparus ne peuvent que lui envier.

Un très grand nombre d'idées toutes faites règnent de nos jours sur la mémoire de la première guerre mondiale et de ses principaux acteurs. Il serait tentant de collecter ces idées dans chaque pays, de la Russie à l'Espagne, en passant par les États d'Europe centrale, et de la Scandinavie à la Grèce. En Allemagne même, des historiens continuent aujourd'hui de reproduire une interprétation négative des " traités de la banlieue parisienne » (Pariser Vorortverträge), à commencer par le diktat de Versailles. Il n'est que de citer ici le livre de l'historien conservateur Hans-Christof Kraus que diffuse depuis quelques années la très officielle "Centrale fédérale pour l'éducation politique ${ }^{1}$ ». On retrouve ici, sans que ce soit toujours dit nettement - mais le titre est clair : Versailles et ses conséquences. La politique extérieure (allemande) entre révisionnisme et entente 1919-1933, l'idée formulée dès 1963 par l'homme politique libéral Theodor Heuss, qui fut le premier président de la République fédérale et qui, sans être nazi, avait voté les pleins pouvoirs au chancelier Hitler en 1933 : «Le mouvement (nazi) n'est pas né à Munich, mais à Versailles ${ }^{2}$. " Encore en 2018, l'historien allemand Holger Afflerbach affirme que la première guerre mondiale aurait pu (dû ?) se terminer par une victoire des empires centraux, si ces derniers n'avaient pas commis trois erreurs : la violation de la neutralité de la Belgique, la guerre sousmarine à outrance et les offensives du printemps $1918^{3}$. L'absurdité de ces conjectures est frappante, on note l'absence du traité de Brest-Litovsk parmi les « erreurs» d'un système intrinsèquement brutal. En 2013-2014 un livre comme Les Somnambules de Christopher Clark a été vendu en Allemagne à plus de 200000 exemplaires $^{4}$, puisqu'il semblait contredire les thèses dominantes de l'historiographie et d'une certaine manière

1. Cet organisme a été créé en 1952 et dépend du ministère fédéral de l'Intérieur. HansChristof Kraus, Versailles und die Folgen. Außenpolitik zwischen Revisionismus und Verständigung 1919-1933, Bonn, Bundeszentrale für politische Bildung, 2014. L'auteur est professeur à l'université de Passau.

2. «Die Geburtsstadt der Bewegung ist nicht München, sondern Versailles» (Theodor Heuss, Erinnerungen 1905-1933 [1963], Frankfurt/Main-Hamburg, Fischer, 1965, p. 170).

3. Holger Afflerbach, Auf Messers Schneide. Wie das Deutsche Reich den Ersten Weltkrieg verlor, München, Beck, 2018. Voir le compte rendu étonnamment bienveillant de Herfried Münkler dans Die Zeit, nº 27, 21 juin 2018, p. 43.

4. Christopher Clark, Die Schlafwandler: Wie Europa in den Ersten Weltkrieg zog, Norbert Juraschitz (trad.), München, Deutsche Verlags-Anstalt, 2013 [traduction de : The Sleepwalkers: How Europe Went to War in 1914, London, Penguin, 2012)]. 
confirmer un certain ressenti allemand quant à la prétendument inutile et humiliante cruauté du traité de Versailles. Cette interprétation n'est pas représentative de toute la recherche allemande et est contredite dans tous les pays concernés par un grand nombre d'historiens qui rappellent certains faits, à savoir que par exemple l'Allemagne a beaucoup moins payé après 1918 que la France après 1871, alors qu'en 1918 les dommages subis par les territoires belges et français étaient éclatants - et qu'en 1871 seule la France avait subi de tels dommages ${ }^{5}$. Était-il par ailleurs monstrueux d'exiger du vaincu qu'il reconnût à l'article 231 du traité de Versailles sa responsabilité ? Peut-être cette formule de Ian Kershaw - postérieure au livre de Christopher Clark dont les conclusions ne se sont nullement imposées dans la communauté des historiens - permetelle de faire le point : « En juillet 1914, au point de rupture, les éléments moteurs de la crise sont l'Allemagne, l'Autriche-Hongrie et la Russie - le rôle de Berlin étant déterminant ${ }^{6}$. " Par ailleurs, malgré le succès du relativisme partial du livre de Christopher Clark, dont les thèses centrales sont d'une part que la guerre est due à un enchaînement mécanique que les acteurs n'ont su maitriser, mais de l'autre que les traités de 1918 reposent sur l'a priori fallacieux que les vaincus étaient coupables, le traité de Versailles et les traités suivants, bien qu'imparfaits à plus d'un titre, ne sauraient être rendus responsables de l'avènement des régimes autoritaires nationalistes et des totalitarismes italien et allemand ni des crimes incommensurables qu'ils causèrent durant la seconde guerre mondiale. Margaret MacMillan, auteur du désormais classique livre Peacemakers. Six Months that Changed the World ${ }^{7}$, ne cesse depuis d'affirmer d'une part que le traité de Versailles n'était pas particulièrement dur, comme dans cette interview allemande, publiée à l'occasion de la traduction allemande tardive, en 2015 seulement $^{8}$, de son livre de 2001 :

5. Nous renvoyons à l'éclairant article de Bruno Cabanes, «Clemenceau vu par Keynes : une réévaluation », dans Sylvie Brodziak et Caroline Fontaine (dir.), Georges Clemenceau et la Grande Guerre, 1906-1929, La Crèche, Geste, 2010, p. 203-215.

6. Ian Kershaw, L'Europe en Enfer : 1914-1949, Aude de Saint-Loup et Pierre-Emmanuel Dauzat (trad.), Paris, Tallandier, 2016, p. 47 [traduction de : To Hell and Back. Europe 1914-1949, London, Penguin, 2015].

7. London, Murray, 2001. Le livre a été traduit en français par André Zavriew : Les artisans de la paix. Comment Lloyd George, Clemenceau et Wilson ont redessiné la carte du monde, Paris, Jean-Claude Lattès, 2006. L'auteur est l'arrière-petite-fille de Lloyd George.

8. Margaret MacMillan, Die Friedensmacher. Wie der Versailler Vertrag die Welt veränderte, Klaus-Dieter Schmidt (trad.), Berlin, Propyläen, 2015. 
Les conditions que l'Empire allemand avait dictées un an auparavant à BrestLitovsk étaient plus dures - la Russie perdait d'énormes portions de son territoire. Pour les réparations, la France a sans doute plus payé à l'Allemagne après 1871 que l'Allemagne après 1919. Après la Seconde Guerre mondiale l'Allemagne fut occupée et divisée. Le traité de Versailles n'a sans doute pas été l'un des plus durs traités de paix jamais imposés à un pays. [...] Répétons-nous : aucune route directe ne conduit de 1919 à 1939. Entre ces deux dates vingt années s'écoulèrent ! Vingt années durant lesquelles des acteurs de toutes sortes prirent des décisions dont la somme pèse plus lourd que ce que Woodrow Wilson, Georges Clemenceau, David Lloyd George et Vittorio Orlando ont décidé en six mois en 1919.

L'idéeselon laquellela paix issue dela première guerre mondialea changé le monde et ne portait pas en soi le germe des meurtrières décennies qui suivirent, peine à s'imposer en Allemagne, comme on l'a vu, puisque l'idée opposée permet, d'une certaine manière, de faire peser la responsabilité des horreurs de l'épisode national-socialiste sur les vainqueurs de 1918 en général et sur le plus vindicatif d'entre eux en particulier, à savoir la France et son président du Conseil, Georges Clemenceau. Le cas particulier de l'Allemagne ne fait cependant qu'illustrer à sa façon le cas général, sinon d'une appréhension globalement négative de la première guerre mondiale et de son résultat, du moins d'un refus de reconnaître que cette guerre a instauré un nouvel ordre européen et mondial qui était riche de promesses. En France même, le traité a été controversé dès sa signature, soit qu'on le jugeât trop clément envers l'Allemagne, comme le pensaient non seulement une certaine droite nationale, du maréchal Foch à l'historien Jacques Bainville, partisan de l'Action française, mais aussi une vaste proportion des anciens combattants ${ }^{10}$, soit qu' on le jugeât au contraire trop dur, voire impérialiste, comme l'affirmait la propagande de la III Internationale et des compagnons de route pacifistes. En 2016, lors de la commémoration de la Bataille de la Somme, une certaine

9. Interview de Margaret MacMillan avec le journaliste Christian Staas, Die Zeit, $\mathrm{n}^{\circ} 46$, 12 novembre 2015, p. 21 : "Die Bedingungen, die das Deutsche Reich ein Jahr zuvor in Brest-Litowsk diktiert hatte, waren härter - Russland verlor gewaltige Teile seines Territoriums. Was die Reparationen angeht, hat Frankreich nach 1871 vermutlich mehr an Deutschland gezahlt als Deutschland nach 1919. Nach dem Zweiten Weltkrieg wurde Deutschland besetzt und geteilt. Der Versailler Vertrag war wohl kaum einer der schwersten Friedensverträge, der je einem Land auferlegt wurde. [...] Noch einmal: Es führt keine direkte Linie von 1919 nach 1939. Dazwischen liegen 20 Jahre! 20 Jahre, in denen verschiedenste Akteure Entscheidungen trafen, die in ihrer Summe schwerer wiegen als das, was Woodrow Wilson, Georges Clemenceau, David Lloyd George und Vittorio Orlando 1919 binnen sechs Monaten beschlossen haben. »

10. Bruno Cabanes, art. cité, p. 213, cite la lettre dans laquelle un soldat non encore 
presse populaire anglaise ultra-conservatrice se contentait d'affirmer qu'en Picardie, c'était l'Empire britannique qui était en jeu : « Les soldats britanniques d'aujourd'hui, qui se tenaient de chaque côté, portaient les médailles d'Afghanistan et d'Irak, d'Irlande du Nord et de Libye. Les médailles des descendants disaient les sacrifices d'une autre ère, quand les hommes qui avaient construit un Empire mouraient pour lui ${ }^{11}$. " Le noyau de vérité de cette citation, c'est que la question de la construction d'une flotte allemande susceptible de mettre en cause la domination britannique des océans est sans doute l'une des principales raisons de l'engagement de Londres dans l'Entente, mais il n'en reste pas moins évident que le résultat de la guerre n'a pas été de stabiliser à long, voire à moyen terme l'Empire britannique - bien au contraire, serait-on tenté de dire.

Le centenaire de la première guerre mondiale a produit une quantité considérable de cérémonies, commémorations, colloques, congrès, rencontres politiques et toutes sortes de publications et de créations artistiques. S'il fallait établir une hiérarchie nationale de l'importance de la mémoire de ce conflit, on pourrait sans peine attribuer l'une des premières places, et probablement la première, à la France, où le 11 novembre s'est affirmé au cours du $\mathrm{xx}^{\mathrm{e}}$ siècle comme la grande journée du souvenir, marquée par des cérémonies dans toutes les communes du pays, tandis qu'en Allemagne cette journée reste connue, depuis le $\mathrm{XIX}^{\mathrm{e}}$ siècle, comme le début du carnaval (à $11 \mathrm{~h} \mathrm{11)}$ ) et que dans les autres pays concernés par la Grande Guerre la plupart du temps, comme du reste en Allemagne, c'est la mémoire de la seconde guerre mondiale, conflit encore plus brutal et plus meurtrier, qui a quasiment supplanté celle de la première. En France même, l'histoire de la première guerre mondiale a été vers la fin du siècle dernier l'objet d'une discussion parfois un peu rude sur le concept de «culture de guerre » proposé par Stéphane Audouin-Rouzeau et Annette Becker et mis en cause par des historiens désireux de faire ressortir les souffrances subies par les soldats au cours du conflit ${ }^{12}$. Vue par un universitaire venant d'une autre discipline, cette

démobilisé commente le traité en juillet 1919 : «Il eût fallu Foch dictateur, faiseur de paix, Foch et non pas Clemenceau. »

11. "Today's British soldiers, who stood on either side, wore medals from Afghanistan and Iraq, Northern Ireland and Libya. The descendants' medals told of the sacrifices of a different era, when the men who had built an empire died for it " (Tom Rowley, "Emotional tributes mark 100th anniversary of the Battle of the Somme», The Daily Telegraph, $1^{\text {er }}$ septembre 2016, https://www.telegraph.co.uk/news/2016/07/01/emotional-tributes-mark-100thanniversary-of-the-battle-of-the-s/ (consulté le 21 septembre 2018).

12. Voir une synthèse de Nicolas Offenstadt, Philippe Olivera, Emmanuelle Picard et 
controverse n'oppose pas des points de vue irréconciliables : s'il est certain qu'en fin de compte l'armée française " a tenu » et a manifesté, même au moment des crises, un incontestable patriotisme (« Notre place est si utile / Que sans nous on prend la pile», dit l'admirable et subversive Chanson de Craonne), il n'en reste pas moins que les hommes et avec eux toute la société ont subi des souffrances telles que le pays ne les a pas oubliées un siècle plus tard. S'il y a une particularité française, elle réside cependant moins dans le bilan des souffrances subies, qui n'excédèrent sans doute pas celles que subirent les soldats et les civils d'autres contrées, que dans le souvenir de ces souffrances entretenu avec système depuis de longues décennies par la société française. D’autres explications de cette pérennité de la mémoire de la première guerre mondiale, liées elles aussi à une « spécificité » française, s’imposent : le fait que les corps des soldats tombés pour la France ont été, dans la mesure du possible, "rapatriés " dans leur commune d'origine, le rite du 11 novembre et, last but not least, le fait que l'on commémore une victoire bien plus éclatante du point de vue français que celle du 8 mai 1945, qui fut précédée par une défaite et plus de quatre dures et humiliantes années d'occupation, la Libération étant immédiatement suivie de presque deux décennies de guerres de décolonisation qui se terminèrent à leur tour, en tout cas d'un point de vue politique, par des défaites sans appel.

À ces explications relatives à l'histoire du pays s'ajoutent selon nous deux autres explications, d'ordre presque psychologique, liées d'une part à l'état d'esprit d'une société moins disposée que d'autres à subir les souffrances liées à la guerre et d'autre part à la conscience, qui s'est estompée au fil des décennies jusqu'à presque disparaître, d'avoir imposé grâce à la victoire de 1918 un nouvel ordre européen appelé à durer. La première de ces explications ne peut être ici que rapidement mentionnée : la société française, consciente d'avoir préservé en 1918 une forme de gouvernement républicain désormais soutenue par une grande majorité de la population, était sans doute moins préparée que des sociétés plus autoritaires ou plus pauvres à subir passivement la guerre, puis à en réprimer le souvenir. La deuxième explication est au centre de cette étude : si l'ennemi par excellence de la France en guerre est l'Allemagne bien plus que l'Autriche-Hongrie, c'est ce dernier État qui disparut, permettant la création de plusieurs nouveaux États-nations reposant sur

Frédéric Rousseau dans Frédéric Rousseau (dir.), Guerres, paix et sociétés, 1911-1946, Neuilly, Atlande, 2004, p. 667-674 (http://www.crid1418.org/espace_scientifique/textes/ culture_de_guerre.htm, consulté le 28 mai 2018). 
le principe de la souveraineté du peuple. Par sa seule existence, l'AutricheHongrie contredisait le projet de stabilisation à long terme du continent européen qui fut porté par les vainqueurs de 1918 dans leur ensemble, et par la France et Clemenceau en particulier. La victoire de 1918 n'était pas seulement celle des nations de l'Entente, elle devait aussi modifier en profondeur le continent européen et empêcher définitivement de nouveaux conflits armés.

Cette conscience d'avoir apporté la paix et l'ordre au continent s'est estompée au cours d'un $\mathrm{xx}^{\mathrm{e}}$ siècle marqué par les totalitarismes, la seconde guerre mondiale, la guerre froide et les guerres de décolonisation. Depuis quelques décennies l'idée proposée par Eric Hobsbawm d'un long XIX ${ }^{\mathrm{e}}$ siècle (1789-1914) suivi d'un court $\mathrm{XX}^{\mathrm{e}}$ siècle (1914-1991) $)^{13} \mathrm{~s}^{\prime}$ est imposée, et avec elle celle de la première guerre mondiale comme le début d'une nouvelle ère. On notera que cette nouvelle ère ne commence pas en 1918, alors qu'est instauré l'ordre issu du conflit mondial, mais en 1914, quand les digues du traditionnel et fragile équilibre européen cèdent. Cela signifie que la crise commence en 1914 et ne se termine qu' avec la chute du mur de Berlin et l'autodissolution de l'URSS en 1991. L'ordre établi par les traités signés de 1919 à 1923 est ramené à la dimension d'un épisode passager que d'autres événements et évolutions semblent vite reléguer dans un passé à jamais révolu. Les deux grandes phases du " court $\mathrm{xx}^{\mathrm{e}}$ siècle " ont en commun de ramener le souvenir français des souffrances et des pertes endurées pendant la première guerre mondiale à celui d'un cruel et inutile sacrifice, un ressenti constatable chez les Français, à commencer par les anciens combattants, dès les années qui suivent le conflit. La première phase, celle qui mène à la seconde guerre mondiale, est marquée par le rapide délitement de l'ordre voulu par les vainqueurs de 1918, en particulier à cause du retrait « isolationniste » des États-Unis d'Amérique et de l'émergence de nouveaux régimes autoritaires en Europe, d'abord l'Union soviétique, ensuite le fascisme et, à l'instar de ce régime, toute une série de pouvoirs autoritaires, voire totalitaires « de droite », créant un rapport de forces défavorable à ce qui reste de la coalition victorieuse de 1918, à savoir les démocraties britannique, française et belge et le fragile

13. Eric Hobsbawm, The Age of Extremes: The Short Twentieth Century, 1914-1991, London, Abacus, 1995 [L'Âge des extrêmes: le court vingtième siècle, 1914-1991, André Leasa (trad.), Bruxelles, Complexe, 2000]. La paternité de la notion de "long XIX ${ }^{e}$ siècle " revient à Ilya Ehrenburg, qui dit que ce siècle dura plus longtemps «que ne le disait le calendrier, il commença en 1789 et se termina en 1914. " (Menschen, Jahre, Leben, München, Kindler, 1962, t. I, p. 21). Voir Gasan Gusejnov, «Long Centuries », Times Literary Supplement, 29 avril 2011. 
royaume de Yougoslavie. Ce qui domine cette phase, c'est l'effondrement de l'ordre voulu par les vainqueurs, une grande impression de faiblesse des démocraties européennes et de gâchis qui rejaillit sur la victoire remportée à la fin de la première guerre mondiale. Cette dernière apparaît alors comme fallacieuse, puisque dans les années qui suivent le conflit le continent européen voit s'étendre l'emprise des régimes autoritaires et, en fin de compte, l'axe Rome-Berlin non seulement défaire dans les années 1930 l'ordre construit par les traités de paix, mais prétendre imposer un " ordre nouveau ». Vu de 1945, le court $\mathrm{xx}^{\mathrm{e}}$ siècle, c'est celui de la naissance et de la défaite du fascisme, du nazisme et de l'expansionnisme nationaliste japonais. La seconde phase remplace après 1945 la brève et violente domination nazie et fasciste du continent européen par celle des deux grandes puissances victorieuses, d'une part l'URSS, qui a joué un rôle militaire de premier plan dans la victoire sur le nazisme, et de l'autre les États-Unis que l'attaque japonaise a définitivement contraints à abandonner un isolationnisme qui dominait l'opinion publique du pays durant l'entre-deux-guerres. L'ère de la guerre froide, qui commence « officiellement » et dans les faits en 1946 (mais le conflit se prépare sans doute bien avant), marque la division de l'Europe et du monde en deux blocs, division d'une certaine manière confirmée par la naissance dans les années 1950 du mouvement des pays non-alignés. Ici, à rebours, on réinterprète la première guerre mondiale comme celle qui a vu naître un nouveau monde, celui du communisme, dont l'emprise s'étend désormais sur une bonne partie du globe et de sa population, notamment depuis que la Chine elle-même est devenue en 1949 une " République populaire ». Au triste constat de l'échec sanglant et inutile de la trompeuse victoire de 1918, qui dominait entre les deux guerres et au constat plus heureux de la défaite des puissances de l'Axe et de la force de la démocratie, s'ajoute l'idée que le grand événement de la première guerre mondiale n'est autre que la révolution d'octobre 1917 qui crée, qu'on l'admire ou non, un système politique qui s'étend inexorablement, car, comme ne manquent pas de le dire non sans quelque raison les propagandistes anticommunistes, si l'on peut citer l'exemple de régimes autoritaires, voire dictatoriaux, qui ont fini par céder à la revendication démocratique, on ne saurait citer (jusqu'en 1989) « aucun exemple de pays communiste ayant effectué une telle transition ${ }^{14} »$. Les deux phases de l'histoire du court $\mathrm{xx}^{\mathrm{e}}$ siècle en

14. Alain Noël et Jean-Philippe Thérien, "L'âge de l'universalité (1945-1980) », dans La gauche et la droite. Un débat sans frontières, Montréal, Presses de l'université de Montréal, 2010, p. 151-193, http://books.openedition.org/pum/4481 (consulté le 8 juin 2018). 
Europe se terminent à chaque fois par une victoire due en grande partie aux États-Unis d'Amérique, contre les totalitarismes de droite en 1945, contre le communisme en 1991. Ces deux phases impliquent le définitif affaiblissement des grandes puissances européennes traditionnelles, le Royaume-Uni, la France et même l'Allemagne, divisée après 1945 en zones d'occupation, puis en 1949 en deux États qui ne font que refléter la bipartition du monde en général et de l'Europe en particulier, et mettent l'accent d'abord sur la guerre froide entre les deux superpuissances - donc, indirectement, sur la naissance de la superpuissance URSS, puis sur la victoire du capitalisme nord-américain sur le communisme à l'échelle européenne et mondiale. Sur cette grande scène mondiale, l'Europe et a fortiori la France semblent condamnées aux rôles de second plan : en somme, l'Europe entamerait en 1914 un suicide collectif qu'elle parachèverait en 1939, laissant la place à l'URSS et aux USA, puis aux seuls USA avec lesquels seule la Chine semble en mesure aujourd'hui de rivaliser bientôt sur le plan politique, comme elle le fait dès maintenant sur le plan économique.

Cette étude tente de prendre le contrepied de ces idées aujourd'hui dominantes en proposant d'interpréter l'ordre établi par les traités qui ont suivi l'armistice du 11 novembre 1918 comme un ordre certes mis en cause par le " court $\mathrm{Xx}^{\mathrm{e}}$ siècle", mais en fin de compte bien plus solide que les divers " ordres nouveaux » que l'on tenta d'imposer au continent européen jusqu'en 1991. Le découpage désormais bien établi pourrait d'abord être corrigé sur un point : ce n'est pas en 1914, mais bien en 1918 que commence le court $\mathrm{XX}^{\mathrm{e}}$ siècle, les années précédentes étant celle de l'agonie du monde du "long XIX ${ }^{e}$ siècle ». Au sortir de la première guerre mondiale naît une Europe nouvelle, sur la base du démantèlement de l'Autriche-Hongrie et de l'Empire ottoman. Cette Europe nouvelle se heurte immédiatement à de puissantes forces contraires, surtout les nationalismes, en Italie, en Allemagne et un peu partout ailleurs. Et le dernier empire, la Russie, subsiste, désireux d'imposer sa domination aux pays européens frontaliers. Ainsi l'URSS n'a fait que perpétuer le dernier empire européen et n'a pas plus créé un prétendu " homme nouveau " qu'un système capable de représenter une durable solution de rechange par rapport au capitalisme.

La disparition de l'Autriche-Hongrie occupe un rang primordial dans l'histoire de l'Europe. S'il est possible de défendre la thèse selon laquelle l'affaiblissement, puis le démantèlement de la double monarchie dès les premiers jours de novembre 1918 contribuèrent grandement à la 
déstabilisation du continent ${ }^{15}$, constat qui s'accompagne souvent d'une condamnation de la trop grande dureté des conditions imposées par le traité de Saint-Germain, on voit moins que la seule survie de cet État dynastique pluriethnique était pour ainsi dire inconcevable dans l'idée d'Europe que la guerre, voulue aussi par les empires centraux, pour s'en tenir à cette formulation prudente, avait fait naître du côté des puissances de l'Entente, notamment en France ${ }^{16}$. Cela ne signifie nullement que l'Autriche-Hongrie ne présentait que des défauts incorrigibles - cette thèse ne résisterait d'ailleurs pas à l'examen des faits -, mais plus simplement qu'elle était incompatible avec le projet d'Europe qui s'imposa au cours du conflit, à défaut d'avoir été conçu auparavant, voire d'avoir fait partie de prétendus buts de guerre des puissances de l'Entente. S'il était impossible de « démanteler » le récent État-nation Allemagne qu'on se contenta pour l'essentiel de priver de ses territoires "non allemands ${ }^{17}$ ", par exemple en restituant la Pologne et en rendant à la France les territoires annexés en 1871, il était devenu impossible de ne pas démanteler l'AutricheHongrie, État par nature pluriethnique. Et, si l'on a pu longtemps penser que la naissance du communisme était l'événement principal de la première guerre mondiale, on peut penser que, du point de vue de l'organisation politique et territoriale du continent européen, c'est bien le démantèlement de l'Autriche-Hongrie qui représente aujourd'hui le grand événement de la période qui s'ouvre à la fin du conflit. Autrement

15. Ce fut le cas de nombreux historiens, à la suite des travaux publiés après la seconde guerre mondiale de Robert A. Kann et Hans Kohn, juifs d'Europe centrale opposés au nationalisme. Voir Jacques Droz, "Présentation ", dans Jacques Droz (dir.), " Les historiens des pays successeurs de l'Autriche-Hongrie ", Austriaca, n 18, 1984, p. 12.

16. Jacques Droz, ibid., p. 11, cite l'historien anglais A. J. P. Taylor, The Habsburg Monarchy 1809-1918: A History of the Austrian Empire and Austria-Hungary [1941], London, Hamish Hamilton, 1949, p. 223-226. Il s'agit d'une liste longue et éclairante des conditions à remplir par les différents acteurs de la double monarchie pour sa survie : cela s'étend des Hongrois qui auraient dû être plus compréhensifs envers les Slaves, et des Allemands, qui auraient dû se détourner de l'Empire allemand, à la personnalité même de François-Ferdinand, "une des pires productions de la Maison Habsbourg ».

17. L'Alsace et une partie de la Lorraine avaient été annexées en 1871 au nom d'un principe d'homogénéité ethnique, mais la France n'avait cessé de considérer ces territoires comme français, étant donné que la population n'avait à aucun moment formé le souhait de rejoindre le territoire d'un État-nation allemand en formation et sous domination prussienne. Cette annexion est à l'origine de la fameuse définition de la nation en tant que "plébiscite de tous les jours » d'Ernest Renan ( Qu'est-ce qu'une nation? », conférence faite en Sorbonne le 11 mars 1882, dans Qu'est-ce qu'une nation?, Joël Roman éd., Paris, Presses Pocket, 1992, p. 37-56). Les autres territoires perdus par l'Allemagne furent donnés à la Belgique, au Danemark et surtout à la Pologne, parfois après des plébiscites locaux, suivis et même précédés de combats, en Haute-Silésie notamment. 
dit, il est tout de même étonnant de lire dans un roman français récent ce passage peut-être ironique, mais fondé sur l'idée qu' " Autrichienne", voire " Autriche » sont des mots dont les collégiens français d'aujourd'hui n'ont pas besoin de s'encombrer la mémoire. À une élève qui est capable de dire ce que signifie le mot " Autrichienne », son professeur de français répond en effet ceci :

Me tournant vers Ming, j'ai dit qu'Autrichienne c'était assez connu en fait, mais bon c'était vraiment un petit pays, qu'on s'en fichait un peu des Autrichiens. Tu connais quand même le pays qui s'appelle l'Autriche, Ming?

- Non.

- Bon ben franchement c'est pas la peine de s'esquinter le cerveau là-dessus, parce qu'en gros, c'est un pays qui n'a aucune importance dans le monde, et pas même en Europe. Est-ce que quelqu'un connaît un Autrichien célèbre?

Aucun doigt levé, c'était plié.

- Voilà, j'vous le disais. Si une bombe rayait l'Autriche de la carte, personne s'en rendrait compte ${ }^{18}$.

Or, il n'est pas possible de réfléchir à l'histoire de la France sans s'intéresser à celle de l'Autriche, c'est-à-dire à celle de la Maison d'Autriche, les Habsbourg, ainsi qu'à un territoire qui a vu naître Adolf Hitler pour qui la France représentait l'Erbfeind, «l'ennemi héréditaire et chauviniste de notre peuple ${ }^{19}$ ", l'Autrichien Hitler ayant une conception raciste de la "germanité ", quel que soit le territoire qu'elle occupe. $\mathrm{Au}$ contraire, on peut considérer la première guerre mondiale comme l'aboutissement du conflit qui a opposé des siècles durant la France monarchique, républicaine, bonapartiste aux différentes configurations politiques de l'Allemagne, du Saint-Empire romain germanique, dominé par la Maison d'Autriche depuis la fin du $\mathrm{Xv}^{\mathrm{e}}$ siècle, jusqu'à l'alliance " nibelungienne ${ }^{20}$ " entre l'Empire des Hohenzollern et l'Autriche-

18. François Bégaudeau, Entre les murs, Paris, Verticales, 2006, p. 176. Cette scène est reproduite dans le film éponyme de Laurent Cantet, qui a obtenu en 2008 la Palme d'or du Festival de Cannes.

19. Voir Adolf Hitler, Mein Kampf, München, NSDAP, 1926, chap. XIII, " Deutsche Bündnispolitik nach dem Kriege ». Il faut noter cependant que le Führer emploie une expression en vogue aussi dans l'historiographie nationaliste française, qui fait un « ennemi héréditaire » de "l'Allemand "- dès l'époque de Clovis que sa conversion rendrait " irrésistible » à la "Gaule romanisée »: voir Jacques Bainville, Histoire de France, Paris, Fayard, 1924, p. 21.

20. Quand on connaît le destin sanglant des personnages de la Chanson des Nibelungen, on 
Hongrie des Habsbourg. On peut même considérer, de ce point de vue, la seconde guerre mondiale comme une tentative d'annihiler l'œuvre politique et territoriale des vainqueurs de la première guerre mondiale, un objectif partiellement atteint malgré la défaite de l'Allemagne, de l'Italie, du Japon et de leurs alliés, puisque la guerre froide divise l'Europe en deux blocs dominés par les superpuissances nord-américaine et russe. On trouvera au passage pleinement confirmé en 1938 le diagnostic posé dès 1929 par Clemenceau à propos des premières années qui suivirent la fin de la guerre : « [...] l'abaissement de la Maison d'Autriche, qui fut un des problèmes de notre vie historique, s'est si bien réalisé par les soins de l'Allemagne, que ce qui reste de l'Empire de François-Joseph en vient à tenter de se fondre, par l'Anschluss, dans la République de Bismarck ${ }^{21}$. » En revanche, depuis la fin de la guerre froide et de cette division, on a assisté à la renaissance de l'Europe forgée contre les Empires centraux par les traités qui suivirent la Grande Guerre.

Si cette analogie avec la vision de la langue en tant que système de signes se différenciant les uns par rapport aux autres est permise, la France s'est construite au fil des siècles en se différenciant par rapport à la grande puissance fédérative ouest- et centre-européenne que représentait au Moyen Âge le Saint-Empire romain. Dès la fin du $\mathrm{Xv}^{\mathrm{e}}$ siècle, la dynastie des Habsbourg incarne une menace quasi existentielle pour le royaume de France, puisqu' elle contrôle les territoires qui l'entourent au nord, à l'est et au sud. Les traités de Westphalie de 1648 permettent à la monarchie française « absolue » d'annexer des territoires du Saint-Empire et d'exporter dans le Saint-Empire son modèle politique, affaiblissant définitivement la position de l'empereur et inaugurant une période de domination partielle, car continuellement contestée, du continent européen. On peut considérer que cette période s'arrête en 1814-1815 avec le congrès de Vienne, qui impose un ordre fondé sur la coopération des grandes dynasties européennes (autrichienne, prussienne et russe) soutenues par le Royaume-Uni, contre le danger que font peser sur elles les "idées nouvelles » et les revendications nationales. La localisation du congrès semble indiquer, sur le plan symbolique, le grand retour des Habsbourg. En fait, contrairement à ce qu'on lit parfois, le congrès de Vienne n'a pas instauré un ordre durable et consensuel. L'éveil des

peut juger plutôt calamiteusel'expression de «fidélité des Nibelungen » (Nibelungentreue), employée le 23 mars 1909 par le chancelier de l'Empire allemand Bernhard von Bülow, dans le cadre de la crise ouverte par l'annexion de la Bosnie-Herzégovine par l'Autriche.

21. Georges Clemenceau, Grandeurs et misères d'une victoire, Paris, Plon, 1930, p. 215. 
nations, le renforcement continu de la revendication démocratique et les traditionnels conflits territoriaux opposant les différentes dynasties, plus vifs que jamais, fragilisent rapidement un équilibre qui n'a jamais pu s'établir dans la durée. Dès 1878, lors de l'organisation à Berlin d'un nouveau congrès, Otto von Bismarck joue le rôle avantageux de l'honnête courtier, tout en signalant cette fois que c'est la Prusse qui domine non seulement le nouvel Empire allemand, mais l'Europe continentale, imposant avec les grandes puissances européennes à la Russie victorieuse de l'Empire ottoman, de douloureuses concessions territoriales : c'est en 1878 que l'on confie à la couronne autrichienne l'administration de la Bosnie-Herzégovine, en renvoyant à une date non précisée une décision définitive sur le statut de ces provinces, et quel'on confirmel'indépendance de la Roumanie, de la Serbie et de la Bulgarie, bien plus petite alors que ce que le libérateur russe et les Bulgares souhaitaient. Autrement dit, c'est aussi ici que se profilent les grandes lignes du conflit en 1914 : d'un côté les vives revendications nationales des "Slaves du sud ", la rivalité entre la Bulgarie et la Roumanie pour la Dobroudja, de l'autre l'impossibilité à long terme pour l'Empire allemand d'être à la fois l'allié de l'AutricheHongrie, qui vise à s'étendre du côté du Sud-Ouest slavophone, et celui de la Russie, qui soutient les Slaves contre la domination autrichienne. On présente souvent le limogeage de Bismarck en 1890 et l'arrimage de Guillaume II à l'Autriche-Hongrie comme la raison principale de l'alliance franco-russe de 1892. En réalité, cette alliance entre deux États que tout semble opposer, une république démocratique et une autocratie autoritaire, ne fait que confirmer le fait que l'équilibre entre les alliances autrichienne et russe était impossible, dès l'époque de Bismarck. Pour les nationaux-socialistes, qui avaient conscience de ce fait, la seule erreur de Guillaume II avait été de prévoir une guerre à mener simultanément sur deux fronts ${ }^{22}$. Que cet équilibre se rompe après le départ de Bismarck ne change rien au fait que l'Allemagne et l'Autriche-Hongrie étaient de toute façon appelées à représenter la force dominante du continent européen, en se passant des alliances britannique et russe grâce auxquelles le congrès de Vienne avait pu un temps construire un équilibre monarchique postrévolutionnaire à l'échelle del'Europe. Y a-t-il eu des «buts de guerre» en 1914 ? Il ne s'agit pas ici de refaire la discussion que déclencha en 1961 le livre de Fritz Fischer Griff nach der Weltmacht, des documents comme

22. Nous pensons ici au film de Wolfgang Liebeneiner, Die Entlassung (1942), dans lequel $a$ posteriori on justifie le pacte de non-agression germano-soviétique d'août 1939. 
le Septemberprogramm du chancelier Bethmann-Hollweg parlent pour eux-mêmes. Il suffira de rappeler deux faits. D'abord le livre Mitteleuropa publié en 1915 par le libéral Friedrich Naumann, qui présentait - non sans raison d'ailleurs - comme modéré un projet qui absorbait la Serbie et la Belgique, et bien entendu toutes les nations de l'Autriche-Hongrie dans un continuum politique et territorial recoupant les frontières dessinées par la première strophe du "Chant des Allemands » de Heinrich August von Fallersleben en 1841, de la Meuse au Niémen, du Belt à l'Adige ${ }^{23}$. En 1940, Theodor Heuss, le futur premier président de la République fédérale d'Allemagne, à une époque où le nazisme semblait triompher sur les plans militaire et politique, rappelait non sans un certain enthousiasme les buts de guerre de Naumann :

Naumann voulait dans le combat mettre l'accent sur un but de guerre allemand - le grand espace continental dominé d'un point de vue politique et économique par les Allemands [...] Nous savons aujourd'hui que ces thèses [...] n'ont pas été rejetées, mais plutôt ajournées. Et qu'elles ne sont pas développées sur le plan de la controverse littéraire, mais sur celui de la décision politique ${ }^{24}$.

Quant au deuxième fait, sur lequel il n'est pas nécessaire de s'appesantir, c'estle traitéde Brest-Litovsk, signéle 3 mars 1918, quetousles observateurs s'accordent à trouver beaucoup plus dur à l'égard de la Russie que ne le fut le traité de Versailles à l'égard de l'Allemagne ${ }^{25}$. Autrement dit, en 19141918 s'est joué le destin de l'Europe face à une alternative relativement claire. En aucun cas le statu quo n'était possible. Comme dans toute crise, c'était un changement décisif qui s'imposait, à moins que l'on considère que la perpétuation de l'Europe des dynasties ait pu déboucher sur autre chose que sur la fameuse Mitteleuropa, c'est-à-dire sur la domination du continent par les Hohenzollern surtout et accessoirement par les Habsbourg, et, parallèlement, sur la relégation des puissances « des

23. Nous renvoyons à notre étude : "L'Europe Centrale, une idée neuve ? ", dans Michel Reffet (dir.), L'Autriche et l'idée d'Europe. Actes du $29^{\circ}$ Congrès de l'AGES, Paris-Dijon, AGES-Éditions universitaires, 1997, p. 359-394.

24. Theodor Heuss, "Mitteleuropa-Literatur ", Europäische Revue, vol. 16, n 2, 1940, p. 627 : « Naumann wollte in dem Machtringen ein deutsches Kriegsziel herausarbeiten - den kontinentalen Großraum unter der politischen und wirtschaftlichen Führung der Deutschen. [...] Wir wissen heute, daß jene Thesen [...] nicht eine Verwerfung, sondern nur eine Vertagung erfahren haben. Und daß sie jetzt ihre Weiterführung nicht in der literarischen Fehde, sondern in der politischen Entscheidung finden.»

25. Interview de Margaret MacMillan, art. cité. Voir aussi la synthèse de Volker Ullrich («Schandfrieden », Die Zeit, nº 7, 8 février 2018, p. 19). 
ailes » ou « des flancs ", à savoir la France et la Russie, à un rôle tout à fait secondaire, tandis que le Royaume-Uni était condamné à se concentrer sur son Empire ou ses relations avec l'Amérique du Nord. Dans le camp de l'Entente, on ne peut distinguer de projet aux contours aussi nets : le Royaume-Uni était surtout motivé par la concurrence impérialiste que représentait un Empire allemand agressif sur le continent, mais aussi sur les océan $\mathrm{s}^{26}$, grâce à une flotte moderne. L'Italie était entrée en guerre en 1915 sur la base du pacte de Londres qui lui offrait la perspective de créer une " frontière naturelle " sur la ligne de partage des eaux en annexant des territoires autrichiens s'étendant du Brenner jusqu'à Trieste. La France, pour sa part, était entraînée dans la guerre par son alliance avec la Russie, pays solidaire de la Serbie menacée d'invasion par l'Autriche-Hongrie qui lui avait déclaré la guerre. Du côté français, la revanche de 1871 et le retour de l'" Alsace-Lorraine ", s'ils apparurent dans la propagande, n'avaient pas motivé une guerre qui résultait d'un système contraignant d'alliances... et, au premier plan, de l'agressivité des empires centraux.

L'entrée en guerre des États-Unis, dont le président Woodrow Wilson proposait une nouvelle forme d'organisation du continent européen sur la base de principes généraux (pas tout à fait généralement appliqués cependant), et la paix séparée conclue par la Russie ont totalement modifié l'issue politique du conflit. Si au départ, de manière quasiment "défensive ", il s'agit d'empêcher la domination du continent par les Empires, à la fin on voit naître un projet cohérent de bouleversement d'un " ordre » traditionnel, qui était fondé sur le pouvoir des grandes dynasties au centre et à l'est du continent. Il fallait briser ces dynasties, il fallait briser les empires. L'évolution politique au sein même du camp de l'Entente a clarifié la situation. Clemenceau a le mieux décrit ce cheminement ( «la force des choses ») qui a conduit du point de vue français aux dispositions des traités de paix signés après l'armistice :

Nous étions partis en alliés des oppresseurs russes de la Pologne, avec les soldats polonais de la Silésie, de la Galicie combattant contre nous. Par l'effondrement de la Russie militaire, la Pologne se trouvait tout à coup libérée, recréée, et voici que dans toute l'Europe, les nationalités relevaient la tête et notre guerre de défense nationale se voyait transformée par la force des choses en guerre de libération.

26. Selon Georges Clemenceau, les Britanniques «n'ignoraient pas cependant que, la Belgique envahie, ils se trouvaient directement menacés dans leurs œuvres vives par une Allemagne qui affirmait que son avenir était sur l'eau » (Grandeurs et misères d'une victoire, op. cit., p. 93). 
L'aspect de la paix se trouvait ainsi totalement changé. Une paix de droit, une Europe de droit, créatrice d'États indépendants dont la puissance militaire s'accroissait de toutes les énergies morales suscitées par le besoin de s'affirmer dans toutes les sphères de la vie internationale ${ }^{27}$.

Au sortir d'une tuerie de quatre ans, provoquée par l'ambition des empires centraux de contrôler le continent européen et d'affaiblir durablement, voire définitivement, la France et la Russie et d'écarter le Royaume-Uni, les vainqueurs ont voulu jeter les bases d'un ordre européen stable, en satisfaisant les revendications nationales des peuples naguère sous domination austro-hongroise, russe et ottomane. Ce projet pouvait rejoindre une certaine propagande anglaise et française, dominante durant le conflit, mais ne répondait pas à un projet conçu dès avant le conflit par les élites politiques. Avant le déclenchement de la première guerre mondiale, les observateurs français s'étonnaient pour la plupart un peu de l'improbable ensemble bariolé que représentait l'Autriche-Hongrie, mais on ne souhaitait pas sa disparition, tant il était difficile de s'imaginer ce par quoi il serait remplacé. Même l'historien Ernest Denis qui fut avec le slaviste Louis Leger durant la guerre l'un des principaux propagandistes de la libération des Slaves opprimés par les Habsbourg, écrivait encore en 1903 que les nationalités slaves " ont un intérêt manifeste au maintien de la monarchie ${ }^{28}$ ", tandis qu'en 1914 le journaliste britannique Henry Wickham Steed, celui-là même qui co-organise du 8 au 11 avril 1918 le congrès qui prône à Rome la liquidation de l'Empire, écrit qu'il n'a perçu, après dix ans de séjour à Vienne, " aucune raison suffisante qui puisse empêcher, en supposant à la dynastie une sagacité moyenne, la monarchie des Habsbourg de conserver sa place légitime dans la communauté européenne ${ }^{29} »$. Pendant la guerre le ton change entièrement, les publications s'en prennent à la monarchie qui s'est inféodée à l'Allemagne et doit donc disparaître, libérant les peuples " opprimés " appelés à fonder leur État-nation. Il n'en reste pas moins que même la censure de la France en guerre

27. Ibid., p. 144.

28. Ernest Denis, La Bohême depuis la Montagne-Blanche, t. II : La renaissance tchèque vers le fédéralisme, Paris, Ernest Leroux, 1903, p. 663. Voir aussi François Genton, « Les historiens de la Troisième République et l'Autriche-Hongrie (1870-1918) ", dans Herta-Luise Ott (dir.), « Penser le pluriculturel en Europe centrale », Chroniques allemandes $\mathrm{n}^{\circ} 11,2006-$ 2007, p. 161-171.

29. Henry Wickham Steed, La monarchie des Habsbourg, Firmin Roz (trad.), Paris, Armand Colin, 1914, p. VIII. 
permet ici un certain pluralisme, puisque paraissent aussi des textes qui approuvent la déclaration de Lloyd George, estimant le 5 janvier 1918 " avec M. Wilson que le démembrement de l'Autriche ne fait pas partie de nos buts de guerre ${ }^{30} »$, ce dernier commentaire se référant aussi aux " 14 points ", fameux discours prononcé trois jours plus tard par le président Woodrow Wilson : le dixième point se contente de demander l'autonomie pour les peuples d'Autriche-Hongrie, un État dont on souhaite expressément garantir la sauvegarde. Toutes ces ouvertures sont condamnées par le système d'alliances dans lequel s'est enfermée la double monarchie. Les tentatives de paix séparée à l'ouest lancées par Charles I ${ }^{\text {er }}$, empereur d'Autriche et roi de Hongrie, notamment en mars 1917 la médiation des princes Xavier et Sixte de Bourbon-Parme, frères de Zita, son épouse, échouent à cause de l'alliance avec l'Empire allemand, dont la politique est alors conduite par le commandement militaire suprême, c'est-à-dire Paul von Hindenburg et Erich Ludendorff, mais aussi à cause de la volonté italienne d'annexer une portion du territoire autrichien. La relative générosité de Lloyd George et de Woodrow Wilson n'est définitivement plus d'actualité au moment du traité de Brest-Litovsk. C'est dans ce contexte que Clemenceau rend publique en avril 1918 la lettre autographe dans laquelle Charles I $^{\text {er }}$ s'engageait le 24 mars 1917 à défendre l'indépendance de la Serbie et les " justes revendications de la France relatives à l'Alsace-Lorraine ", ne faisant ainsi que confirmer que le point de non-retour est atteint depuis la paix séparée avec la Russie. Triste spectacle que celui de Charles Ir démentant alors, contre l'évidence, la révélation de Clemenceau et télégraphiant à Guillaume II le 14 avril 1918 : « Mes canons à l'ouest sont désormais notre réponse ${ }^{31}$. »

30. Le Figaro du 27 janvier 1918, à propos du livre d'Edmond de Fels, L'Entente et le problème autrichien (Paris, Grasset, 1918). La déclaration de Lloyd George, devant le Parlement britannique, date du 5 janvier 1918. Quant au livre de Fels, il exprime un avis devenu minoritaire et les observateurs ne se privent pas de le dire : sa politique « que nous pouvons lire dans son dernier bouquin, celle des aristocrates, est une politique de dupe » : «L'empire d'Autriche consolidé, loin d'être le contrepoids de l'empire d'Allemagne, sera toujours obligé de s'appuyer sur Berlin et nous retrouverons en face de nous l'agglomérat de ses peuples obéissant au mot d'ordre de l'état-major allemand ». Voir aussi une lettre du 18 janvier 1918 d'Étienne de Nalèche, directeur du Journal des Débats, à son ami l'industriel Pierre Lebaudy, https://naleche.hypotheses.org/1490 (consulté le 13 juin 2018).

31. "Unsere weitere Antwort sind meine Kanonen im Westen ", cité d'après Richard von Kralik, Allgemeine Geschichte der Neuesten Zeit von 1815 bis zur Gegenwart, Graz, Styria, 1923, t. VI, p. 550. Le livre de Bernard Michel (La chute de l'Empire austro-hongrois 1916-1918, Paris, Robert Laffont, 1991) reste ici une référence incontournable. 
La logique de Clemenceau, au moment des traités de paix, s'exprime dans son livre-testament Grandeurs et misères d'une victoire et dès le 30 juin 1919, deux jours après la signature du traité de Versailles, dans le discours qu'il prononce à l'Assemblée nationale :

À la seule condition que nous demeurions présents au devoir, le vieil esprit de domination guerrière peut être à jamais terrassé ; le jour est venu où la force et le droit, redoutablement séparés, doivent se rejoindre pour la paix des peuples au labeur. Que l'humanité se lève pour vivre toute sa vie ${ }^{32}$ !

Ce n'est pas le lieu ici de rappeler ce qui a causé l'échec de cette ambitieuse tentative de créer une Europe pacifique où les " peuples au labeur », sous des régimes (implicitement démocratiques) réunissant « la force et le droit ", travailleraient de concert au bien de tous. Il a déjà été fait allusion à la force des nationalismes, en Italie, mais aussi, bien avant 1933 en Allemagne et ailleurs. Une autre cause est le retrait des États-Unis qui n'ont pas honoré la signature apposée aux différents traités de paix, au grand regret de Woodrow Wilson dont le dernier discours, prononcé le 11 novembre 1923, deux jours après le putsch d'Adolf Hitler à Munich, revient avec sévérité sur ce qu'il considère comme une véritable trahison, prophétisant l'inévitable moment où cette erreur " profondément mortifiante » devrait être corrigée :

Les souvenirs stimulants de cette période heureuse de triomphe sont pour nous à jamais gâtés par l'amertume de la honte ressentie devant le fait qu'une fois la victoire remportée, qu'on s'en souvienne, grâce à l'esprit indomptable et aux généreux sacrifices de nos incomparables soldats, nous tournâmes le dos à nos associés et refusâmes de prendre nos responsabilités dans l'administration de la paix ou la consolidation ferme et permanente des résultats d'une guerre remportée au prix de pertes humaines et matérielles terribles, nous retirant dans un maussade et égoïste isolement qui est profondément ignoble, car manifestement lâche et déshonorant.

Que cela reste pour toujours une source de profonde mortification pour nous et, inévitablement, le jour viendra où nous serons contraints par les obligations morales de la liberté et de l'honneur de réparer cette erreur fatale et d'assumer une fois de plus le rôle du courage, du respect de soi et de l'esprit de secours que tous les vrais Américains doivent souhaiter considérer comme notre emploi naturel dans les affaires du monde ${ }^{33}$.

32. Georges Clemenceau, Discours de paix, Paris, Plon, 1938, p. 112-113.

33. The Papers of Woodrow Wilson, vol. 68: April 8, 1922-1924, Arthur S. Link (éd.), Princeton 
À cet isolement nord-américain "maussade et égoïste », il faudrait aussi ajouter l'attitude britannique, qui donne le mauvais rôle à la France et à la Belgique qui tentent en janvier 1923 d'obtenir par l'occupation de la Ruhr une partie des réparations que promettait le traité de Versailles. Clemenceau, en 1929, ne peut s'empêcher de citer la conversation qu'il eut en mars 1921 avec Lloyd George. Si la citation n'est pas exacte, ce que nous ne pouvons pas savoir, elle reflète tout de même l'état des relations entre les deux pays au moment des négociations. À Lloyd George qui lui demande ce qu'il a à lui dire, Clemenceau répond :

- Oui bien, répondis-je, j'ai à vous dire que dès le lendemain de l'armistice, je vous ai trouvé l'ennemi de la France.

- Eh bien, me répondit-il, n'est-ce pas notre politique traditionnelle ${ }^{34}$ ?

Cela dit, l'isolationnisme nord-américain et l'indifférence hostile britannique n'ont fait qu'affaiblir la position des démocraties face au danger qu'ont représenté les nationalismes italien et allemand et le profit qu'ils ont tiré du manque de solidarité entre les États créés sur les décombres des empires démantelés et de la volonté russe de jouer des divisions européennes pour revenir en force.

L'objectif de cette étude est double. Il s'agit d'abord de montrer que le maintien de l'Autriche-Hongrie, encore déclaré comme « but de guerre » par Lloyd George et Woodrow Wilson en janvier 1918, était devenu tout simplement impossible dans les conditions créées par l'alliance « nibelungienne » avec l'Empire allemand, dont l'expression la plus forte fut sans doute le traité de Brest-Litovsk. En second lieu, cette étude vise à montrer que l'ordre politique et territorial établi par les traités qui ont suivi la victoire de l'Entente a été finalement bien plus solide que ce qu'on

University Press, 1993, p. 466 : « ... the stimulating memories of that happy time of triumph are forever marred and embittered for us by the shameful fact that when the victory was won, be it remembered - chiefly by the indomitable spirit and ungrudging sacrifices of our incomparable soldiers - we turned our backs on upon our associates and refused to bear any responsible part in the administration of peace, or the firm and permanent establishment of the results of the war - won at so terrible a cost of life and treasure and withdrew into a sullen and selfish isolation which is deeply ignoble because manifestly cowardly and dishonorable. I This must always be a source of deep mortification to us and we shall inevitably be forced by the moral obligations of freedom and honor to retrieve that fatal error and assume once more the role of courage, self-respect and helpfulness which every true American must wish to regard as our natural part in the affairs of the world. "

34. Georges Clemenceau, Grandeurs et misères d'une victoire, op. cit., p. 93. 
en disait encore dans les dernières décennies du $\mathrm{xx}^{\mathrm{e}}$ siècle, quelques années avant la fin de l'URSS et de son empire européen, notamment en Autriche, l'un des pays où les douleurs consécutives à l'amputation territoriale sont les plus fortes. Plus personne aujourd'hui ne serait tenté de dire : «Retour au futur, c' est-à-dire en route vers l'Europe centrale ! », comme le faisaient encore en 1986 Erhard Busek et Emil Brix ${ }^{35}$. Sans doute sous l'impression des 200000 morts causés par les guerres de Yougoslavie, François Furet parla en 1995 avec mépris du nouvel ordre européen créé après 1918 :

[Les traités multiplient] les États slaves sur les ruines du germanisme vaincu, créant un peu partout, de Varsovie à Prague, et de Bucarest à Belgrade, d'improbables Républiques parlementaires où les bourgeois français croient replanter leurs traditions alors qu'ils exportent leur régime. Plus qu'une paix européenne, les traités de 1919-1920 constituent une révolution européenne. Ils effacent l'histoire de la deuxième moitié du XIX ${ }^{e}$ siècle au profit d'un redécoupage abstrait de petits États multiethniques qui ne reproduisent que les défauts de l'Empire d'Autriche-Hongrie ${ }^{36}$.

Ce " redécoupage abstrait " montre une stabilité remarquable, l'Europe, née après 1918 et détruite par les nationalismes, est revenue après la chute du Mur, à quelques nuances politiques et territoriales près. Même si l'Union européenne connaît des tensions et des conflits politiques en ce moment, la probabilité d'une guerre entre des États regrettant leur grandeur et leur étendue passées, par exemple la Hongrie, et leurs voisins, semble tout de même fort mince, étant donné que dans les conditions actuelles, il est possible de circuler librement d'un pays à l'autre pour faire ses études et travailler et que dans chaque État le droit des minorités est respecté. C'est parce que ces problèmes n'ont pas encore de solution que certains États issus du délitement de l'ex-Yougoslavie ou de l'URSS ne sont pas encore en mesure de s'associer au projet européen. À 26 ans, Winston Churchill dénonça au parlement britannique le danger que faisait courir la démocratie à la paix européenne et le potentiel de violence qu'elle recelait :

35. Erhard Busek et Emil Brix, Projekt Mitteleuropa, Vienne, Überreuter, 1986, p. 171 : «Zurück zur Zukunft - also auf nach Mitteleuropa! » Voir Mitteleuropa. Pour ou contre l'Europe, Gilbert Krebs (éd.), Paris, Presses Sorbonne nouvelle, 1988, avec des textes de Gerard Heimann, Werner Weidenfeld, Erhard Busek, Franz Slawik et François Fejtö (https://books.openedition.org/psn/3473). Après la chute du Mur, Erhard Busek a surtout œuvré pour l'intégration des nouveaux États membres de l'Union européenne.

36. François Furet, Le passé d’une illusion, Paris, Robert Laffont-Calmann-Lévy, 1995, p. 74. 
Mais de nos jours, alors que les masses considérables sont poussées les unes contre les autres et, séparément, chaque individu aigri et fanatisé, quand les ressources de la science et de la civilisation balaient tout ce qui pourrait modérer leur furie - une guerre européenne ne pourra que se terminer par la ruine des vaincus et la dislocation et l'épuisement économiques à peine moins fatals des vainqueurs.

La démocratie est plus vindicative que les cabinets. Les guerres des peuples seront plus terribles que celles des rois ${ }^{37}$.

En réalité, ce qui a causé les guerres les plus meurtrières en Europe, ce fut précisément la contradiction qui opposait l'existence d'empires dynastiques ou dictatoriaux aux revendications démocratique et nationale. On peut même considérer la seconde guerre mondiale comme la terrible résurgence d'une volonté impériale (Hitler n'était-il pas à la tête du Großdeutsches Reich ?) et les guerres qui ont ensanglanté les Balkans jusqu'à la fin du $\mathrm{xx}^{\mathrm{e}}$ siècle comme la conséquence de la dislocation de ces empires : la solution ne peut être qu'une coexistence apaisée de ces territoires dans l'ensemble de type fédératif nouveau, démocratique et attentif au respect des droits de l'homme, que représente l'Union européenne. Par ailleurs, les principes mis en avant lors des « ronflantes déclarations des nouveaux traité ${ }^{38}$ » (par exemple la fondation de la Société des Nations) ouvraient à terme la perspective de l'émancipation des peuples colonisés et de l'engagement généralisé contre le racisme, mais, comme nous le savons, cette perspective était encore lointaine et porteuse de conflits particulièrement violents (la seconde guerre mondiale et les guerres de décolonisation), ce qui n'est pas une raison pour nier que les bases politiques de cette évolution à l'échelle mondiale ont été jetées en 1919. Pour ce qui concerne notre continent, l'Union européenne actuelle pourrait-elle être le début de la réalisation des projets de paix perpétuelle du XviII ${ }^{\mathrm{e}}$ siècle, par exemple celui de l'abbé de Saint-Pierre dont Jean-Jacques Rousseau,

37. «But now, when mighty populations are impelled on each other, each individual severally embittered and inflamed - when the resources of science and civilization sweep away everything that might mitigate their fury - a European war can only end in the ruin of the vanquished and the scarcely less fatal commercial dislocation and exhaustion of the conquerors. Democracy is more vindictive than Cabinets. The wars of peoples will be more terrible than those of kings ", https://winstonchurchill.org/resources/speeches/1901-1914-rising-star/ army-reform/ (consulté le 15 juin 2018).

38. Emmanuelle Sibeud, «1919. Deux conférences pour changer le monde », dans Patrick Boucheron (dir.), Histoire mondiale de la France, Paris, Seuil, 2017, p. 584. 
qui l'admirait, disait qu'il ne pourrait jamais être réalisé ? " Admirons, écrivait Rousseau, un si beau plan, mais consolons-nous de ne pas le voir exécuter ; car cela ne peut se faire que par des moyens violents et redoutables à l'humanité ${ }^{39}$. »

Michel de Certeau, parlant du « lieu » de l'historien, a mis en avant la double fonction de l'historiographie, "narrative » (raconter le passé, l'intégrer par le récit au présent) et " performative » (tirer une leçon du passé pour le présent et l'avenir $)^{40}$. Peut-être cet exemple pris dans l'histoire récente de la Slovénie permet-il d'illustrer cette thèse. En 1989, alors que s'annonçaient la crise yougoslave et le démantèlement de cette fédération, la Slovénie adopta l'hymne national «Zdravljica» (Le toast), poème de Francè Prešeren (1800-1849), publié en 1844, mis en musique en 1905 par Stanko Premrl. Depuis 1991, année de l'indépendance du pays, seule la septième strophe est chantée, ici traduite littéralement :

Que vivent tous les peuples

Qui languissent après le jour

Où partout où va le soleil

Disparaîtra du monde la discorde.

Où tous les humains seront libres

Où ne sera pas un ennemi, mais un voisin celui qui habite de l'autre côté de la frontière ${ }^{41}$.

39. Jean-Jacques Rousseau, "Jugement sur la Paix perpétuelle ", Collection complète des cuvres de J. J. Rousseau, citoyen de Genève, Genève, 1782, t. XII, p. 52.

40. Michel de Certeau, L'écriture de l'histoire, Paris, Gallimard, 2002, p. 140-141.

41. Le titre du poème peut aussi être traduit par «Je lève mon verre ». Voici le texte original de la $7^{\mathrm{e}}$ strophe : "Živé naj vsi narodi, / ki hrepené dočakat' dan, / da, koder sonce hodi, / prepir iz sveta bo pregnan, / da rojak prost bo vsak, /ne vrag, le sosed bo mejak! » Voir également la traduction française dans Francè Prešeren, Les Poésies, Kolja Mićević (trad.), Tatjana Globokar (préf.), Paris, Ésopie, 2013, p. 61 (éd. bilingue). 